\title{
The Relationship between the Etiology of Profound Prelingual Sensorineural Hearing Loss and the Results of Vestibular-Evoked Myogenic Potentials
}

\author{
Alice Andrade Takeuti ${ }^{1}$ Ana Paula Sousa Correa ${ }^{1}$ Elisa Morais Leao ${ }^{1}$ Mariana Lopes Favero ${ }^{1}$ \\ ${ }^{1}$ Division of Education and Rehabilitation of Communication \\ Disorders (Derdic), Speech Therapy Department, Pontifícia \\ Universidade Católica de São Paulo, São Paulo, Brazil \\ Int Arch Otorhinolaryngol 2019;23:1-6.

\begin{abstract}
Address for correspondence Elisa Morais Leao, MD, Otolaryngologist, Divisão de Educação e Reabilitação dos Distúrbios da Comunicação, Departamento de Fonoaudiologia, Pontifícia Universidade Católica de São Paulo, Rua Professora Doutora Neyde Apparecida Sollito, 435, São Paulo, SP, 04022-040, Brazil
\end{abstract} \\ (e-mail: elisamoraisleao@gmail.com; elisaleao@terra.com.br).
}

\begin{abstract}
Keywords

- Vestibular-evoked myogenic potentials

- hearing loss

- prelingual deafness

- deafness

Introduction Cervical vestibular-evoked myogenic potentials (cVEMPs) are biphasic, short latency potentials, which represent the inhibition of the contraction of the sternocleidomastoid muscle (SCM) mediated by the saccule, the inferior vestibular nerve, the vestibular nuclei and the medial vestibular spinal tract.

Objective To evaluate the response of CVEMPs in individuals with profound prelingual bilateral cochlear hearing loss.

Methods A prospective case-control study. A total of 64 volunteers, divided into a study group (31 patients with profound prelingual sensorineural hearing loss) and a control group ( 33 subjects matched for age and gender with psychoacoustic thresholds of $\leq 25 \mathrm{~dB}$ HL between 500 and $8,000 \mathrm{~Hz}$ ) were submitted to the cVEMP exam. The causes of hearing loss were grouped by etiology and the involved period.

Results The subjects of the study group are more likely to present changes in CVEMPs compared to the control group (35.5\% versus $6.1 \%$ respectively; $p=0.003)$, with an odds ratio (OR) of $8.52(p=0.009)$. It means that they had 8.52 -fold higher propensity of presenting altered cVEMP results. There were no statistically significant differences between the latencies, the interamplitude and the asymmetry index. Regarding the etiology, there was a statistically significant difference when the cause was infectious, with an OR of $15.50(p=0.005)$, and when the impairment occurred in the prenatal period, with an OR of $9.86(p=0.009)$.

Conclusion The present study showed abnormalities in the sacculocolic pathway in a considerable portion of individuals with profound prelingual sensorineural hearing loss due to infectious and congenital causes, as revealed by the cVEMP results.
\end{abstract}

\section{Introduction}

The Vestibular-evoked myogenic potential (VEMP) is formed by myogenic responses activated by acoustic, galvanic or bone vibration stimulation, and recorded by surface electromyography. ${ }^{1}$ Colebatch et al first introduced the cervical VEMP (cVEMP), the most commonly used method, which is the assessment of the myogenic activity of the sternocleidomastoid muscle $(\mathrm{SCM})^{2}$ Until its advent, it was impossible to evaluate the otolithic organs. ${ }^{3}$

Cervical vestibular-evoked myogenic potentials is a biphasic, short-latency potential that represents the inhibition of the contraction of the SCM mediated by the saccule. ${ }^{4}$ When a highintensity sound is generated, it stimulates the saccule and, received

December 6, 2017

accepted

March 18, 2018

published online

July 5, 2018
DOI https://doi.org/

10.1055/s-0038-1649491. ISSN 1809-9777.
Copyright $(2019$ by Thieme Revinter

Publicações Ltda, Rio de Janeiro, Brazil
License terms

(요 (1) $\Theta \circledast$ 
consequently, the inferior vestibular nerve (IVN) and its nucleus in the brainstem. The impulses generated by the vestibular nucleus are sent to the SCM ipsilaterally, through the medial vestibulospinal tract, causing the contractile inhibition of its muscular fibers. ${ }^{3}$ The electrical representation of this reflex arc consists of four distinct waves named P1 or P13, $\mathrm{N} 1$ or N23, N34 and P44, due to their respective latencies (13 $\mathrm{ms}$ and $23 \mathrm{~ms}$; and $34 \mathrm{~ms}$ and $44 \mathrm{~ms}$ respectively). The waves N34 and P44 are inconsistent and considered clinically insignificant because the possibility of a non-vestibular origin. ${ }^{4}$

The ocular VEMP (oVEMP) is a recent variant technique with utricular origin. The generation of this potential is mediated by the vestibulo-ocular reflex (VOR) pathway. After the activation of the utricle, the acoustic stimulus is transmitted by the superior vestibular nerve to the medial longitudinal fasciculus (where the decussation occurs), and ends in the oculomotor nucleus and in the ocular nerve, generating a three-phase myogenic potential with a negative peak (N10), a positive peak (P14) and another negative peak in $\sim 23 \mathrm{~ms}^{3}$

The VEMP has several characteristics favorable to its use: it is an objective, non-invasive, easy-to-perform, low-cost, fast, and non-discomforting exam. ${ }^{1}$ Variations of its parameters have been demonstrated in a many different conditions, such as: Ménière disease, benign paroxysmal postural vertigo, acute vestibular neuritis, pontocerebellar angle tumors, acoustic trauma, noise-induced hearing loss, central nervous system disorders and gentamicin therapy. ${ }^{4}$

Studies show that the absence of hearing does not make it impossible to obtain VEMP measurements. However, it is well known that anatomically and phylogenetically, the vestibular and cochlear receptors, the semicircular canals and the otolithic organs are closely related. They share the continuous membranous labyrinth of the inner ear, and function by means of very similar receptor cells. In recent years, there has been a growing awareness of vestibular dysfunction in hearing impaired children, with studies demonstrating the presence of peripheral vestibular deficit in these patients with severe to profound sensorineural hearing loss. ${ }^{5}$ In contrast, there is little interest in vestibular dysfunction in adults with profound sensorineural hearing loss, possibly because those individuals already have central compensation.

There are no studies with adults with deafness that associate the etiology of hearing loss with VEMP results.

Therefore, the hypothesis of the present study is that individuals with profound bilateral sensorineural and prelingual hearing loss show changes along the vestibular pathway measured by the cVEMPs. The objective is to evaluate the results of cVEMPs in individuals with profound prelingual sensorineural hearing loss and to correlate them with the etiology.

\section{Method}

A case-control study approved by the Ethics and Research Committee of our institution under report number 912.452, which was conducted with 64 volunteers who signed the
Free and Informed Consent Form, and were divided into 2 groups.

1. Study Group (SG), composed of 31 individuals with deafness. The inclusion criterion was individuals with deep prelingual sensorineural hearing loss, confirmed by tonal audiometry, and a psychoacoustic threshold of $\geq 90 \mathrm{~dB}$ $\mathrm{HL}$ in the frequencies between $500 \mathrm{~Hz}$ and 8,000 $\mathrm{Hz}$.

2. Control group (CG), consisting of 33 individuals matched by age and gender, and with a psychoacoustic threshold of $\leq 25 \mathrm{~dB} \mathrm{HL}$ in the frequencies between $500 \mathrm{~Hz}$ and $8,000 \mathrm{~Hz}$.

The exclusion criteria for both groups were complaint of dizziness; previous history of congenital or acquired middle ear pathology; and neurological diseases (tumoral, diffuse lesions, demyelinating diseases or functional alterations).

The participants answered a questionnaire containing information about the etiology and age of onset of the hearing loss. With the participant in a sitting position, negative electrodes were placed on the sternal furcula, the positive ones in the cervical region on the middle third of the sternocleidomastoid muscles (SCMs), and the ground electrode in the frontal region after preparation of the skin with alcohol and abrasive paper.

The cVEMP was recorded using Intelligent Hearing System (two-channel Smart EP windows USB version 3.91) with insert earphones ER-3. Tone burst stimuli of $500 \mathrm{~Hz}$ with rarefied polarity, presentation rate of 4.3 stimuli per second, with an intensity of $99 \mathrm{~dB}$ HL, trapezoidal envelope, rise-fall time of $2,000 \mu \mathrm{g}$, and a plateau of 3,000 $\mu \mathrm{g}$ were presented through the insertion of earbuds in both ears simultaneously. A $10-\mathrm{Hz}$ high-pass filter and a $1,500-\mathrm{Hz}$ low-pass filter were used. The potentials were captured ipsilaterally in a window of $51.2 \mathrm{~ms}$, totaling an average of 150 stimuli. The characteristics of the stimulus to elicit cVEMPs were used according to the standard proposed by the International Guideline for the clinical application of "VEMPs. ${ }^{6}$

The seated patient was instructed to rotate his/her head at $\sim 90^{\circ}$ from the vertical plane to the sides and to perform adequate force to keep the SCM contracted, contralaterally to the source of the sound stimulus.

\section{Results}

Descriptive data analysis was performed by means of absolute and relative frequencies, central tendency measures (mean and median) and dispersion (standard deviation [SD], minimum and maximum values).

Cases and controls were paired by gender and age, with no statistically significant difference. When comparing both groups for the gender variable, the Chi-squared test for association was applied. As for age, there was no adherence to the normal curve analyzed by the Kolmogorov-Smirnov test, since it the nonparametric Mann-Whitney test was used, as well as for the other quantitative variables.

To verify the association between the "altered" independent variable and the case and control groups, we used the 
Table 1 Distribution of pairing variables, according to groups

\begin{tabular}{|c|c|c|c|c|c|}
\hline \multirow[t]{2}{*}{ Variable } & \multicolumn{2}{|c|}{ Case } & \multicolumn{2}{|c|}{ Control } & \multirow[t]{2}{*}{$p\left(x^{2}\right)$} \\
\hline & $\mathrm{n}$ & $(\%)$ & $n$ & $(\%)$ & \\
\hline \multicolumn{6}{|l|}{ Gender } \\
\hline Male & 16 & $(51.6)$ & 15 & $(45.5)$ & 0.622 \\
\hline Female & 15 & $(46.4)$ & 18 & $(54.4)$ & \\
\hline Total & 31 & $(100.0)$ & 33 & $(100.0)$ & \\
\hline Age & & & & & $p^{*}$ \\
\hline Mean & \multicolumn{2}{|c|}{23.87} & \multicolumn{2}{|c|}{25.64} & 0.105 \\
\hline (SD) & \multicolumn{2}{|c|}{$(7.26)$} & \multicolumn{2}{|c|}{$(6.64)$} & \\
\hline Median & \multicolumn{2}{|c|}{20.00} & \multicolumn{2}{|c|}{24.00} & \\
\hline Minimum & \multicolumn{2}{|l|}{15} & \multicolumn{2}{|l|}{15} & \\
\hline Maximum & \multicolumn{2}{|l|}{44} & \multicolumn{2}{|l|}{42} & \\
\hline
\end{tabular}

Abbreviations: $\mathrm{X}^{2}$, Chi-squared test; SD, standard deviation. Note: *Mann-Whitney test.

Chi-squared test and the conditional univariate logistic regression analysis to express the odds ratio (OR).

A descriptive level of $5 \%(p<0.05)$ was assumed for statistical significance. The data was entered in the Microsoft Excel software (Microsoft Corporation, Redmond, WA, USA), and analyzed using the Statistical Package for the Social sciences (SPSS, IBM Corp, Armonk, NY, USA) software, version 22.0 for Windows.

A total of 31 cases and 33 controls were analyzed. - Table 1 demonstrates that there was no statistically significant difference between the groups for the paired variables, gender and age.

It is possible to observe on - Table 2 that in the comparison between cases and controls, according to the altered independent VEMP variable response, that is, the absence of response and asymmetry index $>35 \%$, there was a statistically significant difference between the groups. The individuals in the case group were more likely to present this change when compared to the control subjects (35.5\% versus $6.1 \%$ respectively; $p=0.003)$, with an OR of $8.52(p=0.009)$. In other words, individuals in the case group have an 8.52fold higher chance of presenting altered results in cVEMPs.

As shown in - Table 3, the analyzed variables, wave peak latencies (ms), the interamplitude between the positive wave peak and the negative wave valley $(\mu \mathrm{V})$ and the asymmetry index (\%) did not present statistically significant differences between cases and controls $(p>0.05)$.

Five patients were unable to report the cause and age of their hearing loss. Therefore, an adjustment was made to the number of individuals in the sample for the statistical analysis of the etiologies of hearing loss. -Table 4 shows that there was no statistically significant difference between the groups. - Table 5 shows that the congenital rubella and genetic/hereditary etiologies had a higher percentage of occurrence (27\%). Regarding the age of the onset of deafness, the overall mean age was of 8.95 months $(S D=14.80)$, median of 0.67 , ranging from less than 1 month to 36 months (-Table 6).

As shown in - Table 7, there was a statistically significant association between etiology and cVEMP alteration. Based on the OR analysis, there is a 15.50 -fold ( $p=0.005)$ higher chance of a patient with infectious etiology to exhibit alterations in the exam when compared to the controls.

- Table 8 indicates that there was a statistically significant association between the period of acquired deafness and the result of the modified cVEMP. Based on the OR analysis, there is a $9.86(p=0.009)$ chance of a patient with congenital hearing loss to present alterations in cVEMPs when compared to the controls.

\section{Discussion}

Recent studies suggest that the cVEMP is a vestibulocolic reflex (VCR) whose afferent pathway begins in the acoustically sensitive cells in the saccule. It accesses, in addition to the saccule, the inferior vestibular nerve and the medial vestibulospinal tract. If individuals with severe to profound hearing loss really present an impairment of the vestibular system (according to the hypothesis of the present study), the cVEMP becomes very useful in the identification of damages along the sacculo pathway of these individuals. In addition, it is an affordable, fast, low-cost and well tolerated method. ${ }^{1}$

Several studies have demonstrated the existence of saccular dysfunction in individuals with hearing loss, based on cVEMP results. ${ }^{4}$ The most predominant deviations in the literature are a significant reduction in the interpeak P1/N1 amplitude and the absence of responses in hearing-impaired individuals. Generally, no significant difference was observed for the P1 and N1 latencies in these patients. 3,6

Our research has also found a considerable rate of cVEMP alterations in individuals with profound prelingual hearing

Table 2 Chi-squared association analysis and univariate binary logistic regression, second change in cVEMPs

\begin{tabular}{|c|c|c|c|c|c|c|}
\hline \multirow[t]{3}{*}{ Variable } & \multicolumn{5}{|l|}{ Group } & \multirow{3}{*}{$p$} \\
\hline & Case & Control & \multirow[t]{2}{*}{$p\left(x^{2}\right)$} & \multirow[t]{2}{*}{ OR } & \multirow[t]{2}{*}{$95 \% \mathrm{Cl}$} & \\
\hline & n (\%) & n (\%) & & & & \\
\hline \multicolumn{7}{|c|}{ Alteration } \\
\hline No & $20(64.5)$ & 31 (93.9) & 0.003 & 1.0 & & \multirow[t]{2}{*}{0.009} \\
\hline Yes & $11(35.5)$ & $2(6.1)$ & & 8.52 & $1.7-42.6$ & \\
\hline
\end{tabular}

Abbreviations: $x^{2}$, Chi-squared test; $95 \% \mathrm{Cl}$, 95\% confidence interval; OR, odds ratio; cVEMPs, cervical vestibular-evoked myogenic potentials. 
4 Etiology of Profound Prelingual Sensorineural Hearing Loss and VEMP Takeuti et al.

Table 3 Qualitative analysis between groups, according to markers of hearing loss

\begin{tabular}{|c|c|c|c|c|c|c|c|c|c|}
\hline \multirow[t]{3}{*}{ Variable } & \multicolumn{8}{|c|}{ Group } & \multirow[t]{3}{*}{$p^{*}$} \\
\hline & \multicolumn{4}{|c|}{ Case } & \multicolumn{4}{|c|}{ Control } & \\
\hline & $\mathrm{n}$ & $\bar{X}(S D)$ & median & Min. - Max. & $n$ & $\bar{X}(S D)$ & median & Min. - Max. & \\
\hline P13 RE (ms) & 31 & $10.31(7.39)$ & 13.60 & $0.0-18.9$ & 33 & $16.00(3.17)$ & 14.60 & $12.4-26,1$ & 0.008 \\
\hline N23 RE (ms) & 31 & $15.57(11.11)$ & 21.10 & $0.0-27.8$ & 33 & $23.47(3.25)$ & 22.90 & $17.8-33.1$ & 0.024 \\
\hline P13 LE (ms) & 31 & $10.36(7.41)$ & 14.40 & $0.0-20.1$ & 33 & $15.62(1.95)$ & 15.50 & $12.7-19.9$ & 0.013 \\
\hline $\mathrm{N} 23 \mathrm{LE}(\mathrm{ms})$ & 31 & $15.63(11.17)$ & 20.80 & $0.0-28.4$ & 33 & $23.45(1.79)$ & 23.40 & $20.1-27.0$ & 0.012 \\
\hline
\end{tabular}

Abbreviations: $\bar{X}$, mean; LE, left ear; RE, right ear; Max., maximum; Min., minimum; SD, standard deviation.

Note: *Mann-Whitney test.

Table 4 Distribution of demographic variables, according to groups

\begin{tabular}{|l|l|l|l|l|l|}
\hline \multirow{2}{*}{ Variable } & \multicolumn{2}{l|}{ Case } & \multicolumn{2}{l|}{ Control } & \multirow{2}{*}{$\boldsymbol{p}\left(\mathbf{X}^{2}\right)$} \\
\cline { 2 - 5 } & $\mathbf{n}$ & $\mathbf{( \% )}$ & $\mathbf{n}$ & $\mathbf{( \% )}$ & \\
\hline Sex & 16 & $(61.5)$ & 15 & $(45.5)$ & 0.219 \\
\hline Male & 10 & $(38.5)$ & 18 & $(54.4)$ & \\
\hline Female & 26 & $(100.0)$ & 33 & $(100.0)$ & \\
\hline Total & 26 & & & \multirow{2}{*}{$\boldsymbol{p}^{*}$} \\
\hline Age (months) & & 25.64 & 0.185 \\
\hline Average & 24.31 & $(6.64)$ & \\
\hline (SD) & $(7.62)$ & 24.00 & \\
\hline Median & 20.50 & 15 & \\
\hline Minimum & 15 & 42 & \\
\hline Maximum & 44 & \multicolumn{2}{|l|}{15} & \\
\hline
\end{tabular}

Abbreviations: $\mathrm{x}^{2}$, Chi-squared test; $\mathrm{SD}$, standard deviation. Note: *Mann-Whitney test.

Table 5 Number and percentage of patients, according to etiology (case group)

\begin{tabular}{|l|l|l|l|}
\hline Variable & Category & $\mathbf{n}$ & $\mathbf{( \% )}$ \\
\hline Etiology & Idiopathic etiology & 6 & $(23.0)$ \\
\hline & Genetics & 7 & $(27.0)$ \\
\hline & Congenital rubella & 7 & $(27.0)$ \\
\hline & Congenital syphilis & 1 & $(3.8)$ \\
\hline & Ototoxicity & 2 & $(7.7)$ \\
\hline & Prematurity & 1 & $(3.8)$ \\
\hline Total & Meningitis & 2 & $(7.7)$ \\
\hline
\end{tabular}

loss. The most significant one was the absence of response in the individuals in the experimental group (35.5\%) when compared to the control subjects (6.1\%), which was statistically significant $(p=0.003)$. Furthermore, the individuals in the case group had an 8.52-fold greater chance of having altered results in relation to the control group (OR; $p=0.009$ ). In the present study, all the individuals in the case group presented absence of responses as altered results (absence of waves). Therefore, there was no reduction in the interpeak P1/N1 amplitude. This result ratifies the current knowledge that the saccule and the cochlea, by sharing the same membranous labyrinth, have great similarity in the ultrastructure of the vestibular and cochlear hair cells. ${ }^{5}$ In addition, the anatomical proximity between the saccule and the afferent system of acoustic energy in the inner ear, combined with the common arterial blood supply of the cochlea and vestibular organs through the same terminal artery, suggest the possibility of deterioration of the vestibule, especially of the saccule, due to the same factors that are damaging to the cochlea (whether of congenital or acquired etiology). ${ }^{7,8}$

It is possible that the genesis of hearing loss is related to the degree of impairment of the pathway investigated by cVEMPs, explaining the fact that individuals with similar hearing loss have different cVEMP results. In our research, the congenital rubella and genetic/hereditary etiologies were the most prevalent. Pre or postnatal infectious causes (rubella, syphilis and meningitis) had a statistically significant association with cVEMP change, with a 15.50 -fold increased chance of altered cVEMPs in relation to the controls. When the etiologies were grouped into congenital and postnatal, we found that intrauterine causes are 9.86 times more likely to provoke altered cVEMPs than controls. Few research papers in the literature relate the findings of VEMPs with the various causes of prelingual hearing loss. Zagrolski detected cVEMP alterations in children with congenital cytomegalovirus infection at birth. ${ }^{9}$ A possible hypothesis for congenital hearing loss to present more changes in the sacculo-colic pathway would be the early involvement, during the embryonic stage, of the cells of the cochlea and the vestibule.

Concerning infectious causes, an aggression by viruses and bacteria to the hair cells, the organ of Corti and the tectonic membrane, in addition to an inflammation of the auditory nerve, are thought to occur. But the exact mechanism remains unknown. These individuals may develop late hydrops later in life, manifested by vestibular symptoms, but without auditory symptoms due to hearing loss. Zagólski, ${ }^{9}$ in his research on infants affected with congenital cytomegalovirus and congenital rubella, found a greater alteration of the vestibular pathway in patients with higher auditory thresholds. In the present study, we have noticed that the change in VEMP outcome is 
Table 6 Descriptive analysis of the age that deafness occurred (cases of congenital etiology and prematurity were excluded)

\begin{tabular}{|c|c|c|c|c|c|}
\hline Variable & $\mathrm{n}$ & $\bar{X}(S D)$ & median & Minimum & maximum \\
\hline \multicolumn{6}{|l|}{ General* } \\
\hline Age (months) & 7 & $11.31(14.72)$ & 0.67 & 0.08 & 36.00 \\
\hline \multicolumn{6}{|c|}{ Function of the inferior vestibular nerve } \\
\hline Age - unaltered group & 6 & $10.19(15.80)$ & 0.46 & 0.08 & 36.00 \\
\hline Age - altered group & 1 & $18.00(-)$ & 18.00 & 18.00 & 18.00 \\
\hline
\end{tabular}

Abbreviations: $\bar{X}$, mean; SD, standard deviation.

Note: *Two cases were ignored, and both were of idiopathic etiology.

Table 7 Analysis of association by the Chi-Squared test and univariate binary logistic regression, according to the presence or not of etiology in the alteration of the cVEMPs

\begin{tabular}{|c|c|c|c|c|c|c|}
\hline \multirow[t]{3}{*}{ Variable } & \multicolumn{5}{|c|}{ Function of the inferior vestibular nerve } & \multirow[t]{3}{*}{$p$} \\
\hline & Unaltered & Altered & \multirow[t]{2}{*}{$p\left(x^{2}\right)$} & \multirow[t]{2}{*}{$\mathrm{OR}^{*}$} & \multirow[t]{2}{*}{$95 \% \mathrm{Cl}$} & \\
\hline & n (\%) & n (\%) & & & & \\
\hline \multicolumn{7}{|l|}{ Etiology } \\
\hline No disease & $31(93.9)$ & $2(6.2)$ & 0.013 & 1.0 & & \\
\hline Genetics & $6(85.7)$ & $1(14.3)$ & & 2.58 & $0.20-33.24$ & 0.467 \\
\hline Infectious $^{*}$ & $5(50.0)$ & $5(50.0)$ & & 15.50 & $2.34-102.85$ & 0.005 \\
\hline Others $^{\S}$ & $7(77.7)$ & $2(22.2)$ & & 4.43 & $0.53-37.07$ & 0.170 \\
\hline Total & $49(83.1)$ & $10(16.9)$ & & & & \\
\hline
\end{tabular}

Abbreviations: $x^{2}$, Chi-squared test; $95 \% \mathrm{Cl}$, 95\% confidence interval; cVEMPs, cervical vestibular evoked myogenic potentials; OR, odds ratio. Notes: ${ }^{*}$ Infectious means meningitis, rubella and syphilis; ${ }^{\S}$ other, idiopathic, prematurity and ototoxicity.

Table 8 Analysis of association by the Chi-squared test and univariate binary logistic regression, according to the acquisition of deafness and alteration in cVEMPs

\begin{tabular}{|l|l|l|l|l|l|l|}
\hline \multirow{2}{*}{ Deafness } & \multicolumn{3}{|l|}{ Function of the inferior vestibular nerve } & \multirow{2}{*}{$\boldsymbol{p}$} \\
\cline { 2 - 6 } & unaltered & Altered & $\boldsymbol{p}\left(\mathrm{x}^{2}\right)$ & OR & $95 \% \mathrm{Cl}$ & \\
\cline { 2 - 6 } & $\mathbf{n}(\%)$ & $\mathbf{n}(\%)$ & & & & \\
\hline No disease & $31(93.9)$ & $2(6.1)$ & 0.011 & 1.0 & & \\
\hline Congenital & $9(56.25)$ & $7(43.75)$ & & 9.86 & $1.78-54.83$ & 0.009 \\
\hline Postnatal & $9(90.0)$ & $1(10.0)$ & & 2.21 & $0.18-27.98$ & 0.539 \\
\hline Total & $49(83.1)$ & $10(16.9)$ & & & & \\
\hline
\end{tabular}

Abbreviations: $x^{2}$, Chi-squared test; $95 \% \mathrm{Cl}$, 95\% confidence interval; cVEMPs, cervical vestibular evoked myogenic potentials; OR, odds ratio.

probably more related to the etiology than to the degree of hearing loss alone, since all patients had thresholds below $90 \mathrm{~dB}$ HL.

Another issue to be addressed is that the genetic/hereditary cause may be more frequent in our sample, since there is a possibility that patients classified as idiopathic may actually have an unidentified genetic cause. Difficult access to genetic testing makes this diagnosis difficult.

There are no standard values of normality in relation to wave latency in the literature; therefore, the latency values of the control group serve as parameters for the study group. The present study, similarly to most studies, found no statistically significant difference in relation to P1 and N1 wave latencies bilaterally between the two groups.
In contrast to other studies, ${ }^{3,5,8}$ there was no statistically significant difference between the groups in relation to the interamplitude between the peak of the positive wave and the valley of the negative wave $(\mu \mathrm{V})$, and the values found are within the range of normality, since the reference value for the asymmetry index adopted in the present study was of up to $35 \%$.

The absence of signs or symptoms related to a peripheral vestibular disorder in adolescents and adults of the experimental group with altered cVEMPs could be due to the contribution of the other systems to the maintenance of the body's balance (visual and somatosensory), linked to the effect of neuroplasticity and mechanisms of central compensation. ${ }^{8}$ Another hypothesis is that saccular dysfunction alone is not sufficient to cause symptoms. ${ }^{5}$ However, during the early 
developmental phase of childhood, the vestibular deficit may impair the integration process of sensory stimuli critical to the normal development of motor coordination and locomotion. The loss of vestibular function places the child at risk of significant impairment of vestibulo-ocular interaction during normal activity and of balance maintenance in dark environments. ${ }^{5}$ Hyporeflexia and reflexes in caloric testing ranged from 20 to $40 \%$ in children with deafness, and utricular hypofunction was present in $20 \%$ of these patients. ${ }^{10}$ The search for vestibular alterations in children with deafness is not routinely performed, just as vestibular symptoms are often not identified by the professionals who accompany them. It is necessary that vestibular disorders be considered a differential diagnosis for children with psychic, behavioral, motor development, and language alterations. ${ }^{11}$

Adult individuals with profound deafness usually do not have vestibular complaints due to central compensation, which occurs around 9 years of age, and are mainly aided by vision and proprioception, with a reorganization of the cortical sensorial regions. ${ }^{12}$ However, with aging, these senses become naturally hypofunctional. Consequently, the risk of falling (which is the main cause of external death in elderly people) increases. As a preventive method, it is crucial to have these individuals rehabilitated as early as possible.

Therefore, clinical assessments of the vestibular system in conjunction with electrophysiological tests are necessary to detect the functional effects of vestibular deficiency in individuals with profound deafness. Furthermore, it is important to keep in mind that the analysis of cVEMPs should consider that the alterations may be due to the etiology of hearing loss and not necessarily to a sacculocolic pathway indicating vestibular dysfunction.

\section{Conclusion}

The present study has demonstrated anomalies in the sacculecochlear pathway in a considerable number of individuals with profound prelingual sensorineural hearing loss due to infectious and congenital causes revealed by cVEMPs results.

\section{References}

1 Felipe L, Kingma H, Gonçalves DU. Potencial evocado miogênico vestibular. Arq Int Otorrinolaringol 2012;16(1):103-107. Doi: 10.7162/S1809-48722012000100015

2 Colebatch JG, Halmagyi GM, Skuse NF. Myogenic potentials generated by a click-evoked vestibulocollic reflex. J Neurol Neurosurg Psychiatry 1994;57(02):190-197. Doi: 10.1136/jnnp.57.2.190

3 Bansal S, Sahni S, Sinha S. Cervical and ocular vestibular evoked myogenic potentials in individuals with severe to profound hearing loss. J Hear Sci 2013;3(04):56-63http://search.ebscohost.com/login.aspx?direct $=$ true\&profile $=$ ehost\&scope $=$ site \&au thtype $=$ crawler\&jrnl $=2083389 X \& A N=95785356 \& \mathrm{~h}=\mathrm{jtm} \% 2 \mathrm{~F} 69$ \%2B1shVu7dTEro6hLA5HxrnjjqieU\%2FDbfA7T\%2Bvv4ze8fdiXXM zL2rC5BA0Tio8NEsciuLBlagdOPuz2eCw\%3D\%3D\&crl=c Accessed May 13, 2014

4 Mudduwa R, Kara N, Whelan D, Banerjee A. Vestibular evoked myogenic potentials: review. J Laryngol Otol 2010;124(10): 1043-1050. Doi: 10.1017/S0022215110001234

5 Singh S, Gupta RK, Kumar P. Vestibular evoked myogenic potentials in children with sensorineural hearing loss. Int J Pediatr Otorhinolaryngol 2012;76(09):1308-1311. Doi: 10.1016/j.jporl.2012.05.025

6 Papathanasiou ES, Murofushi T, Akin FW, Colebatch JG. International guidelines for the clinical application of cervical vestibular evoked myogenic potentials: an expert consensus report. Clin Neurophysiol 2014;125(04):658-666. Doi: 10.1016/j.clinph.2013.11.042

7 Khan F, Balraj A, Lepcha A. Vestibular evoked myogenic potential in sudden sensorineural hearing loss. Indian J Otol 2013;19:55. Doi: $10.4103 / 0971-7749.113504$

8 Sazgar AA, Dortaj V, Akrami K, Akrami S, Karimi Yazdi AR. Saccular damage in patients with high-frequency sensorineural hearing loss. Eur Arch Otorhinolaryngol 2006;263(07):608-613. Doi: 10.1007/s00405-006-0038-6

9 Zagólski O. Vestibular-evoked myogenic potentials and caloric stimulation in infants with congenital cytomegalovirus infection. J Laryngol Otol 2008;122(06):574-579. Doi: 10.1017/ S0022215107000412

10 Tribukait A, Brantberg K, Bergenius J. Function of semicircular canals, utricles and saccules in deaf children. Acta Otolaryngol 2004;124(01):41-48. Doi: 10.1080/00016480310002113

11 Clark DL, Arnold LE, Crowl L, et al. Vestibular Stimulation for ADHD: randomized controlled trial of Comprehensive Motion Apparatus. J Atten Disord 2008;11(05):599-611

12 Nakajima Y, Kaga K, Takekoshi H, Sakuraba K. Evaluation of vestibular and dynamic visual acuity in adults with congenital deafness. Percept Mot Skills 2012;115(02):503-511. Doi: 10.2466/15.06.25.PMS.115.5.503-511 